Check for updates

Cite this: RSC Adv., 2018, 8, 32377

Received 10th July 2018

Accepted 5th September 2018

DOI: $10.1039 / \mathrm{c} 8 \mathrm{ra05868g}$

rsc.li/rsc-advances

\section{Revealing the deformation mechanism of amorphous polyethylene subjected to cycle loading via molecular dynamics simulations}

\begin{abstract}
Qihong Fang, ${ }^{a}$ Yuanyuan Tian, ${ }^{\mathrm{a}}$ Hong $\mathrm{Wu}^{* \mathrm{bcd}}$ and Jia Li (D) *a
Despite tremendous efforts being devoted to the study of the deformation behavior of polyethylene, the deformation mechanism of an amorphous polyethylene polymer under cycle shear-loading remains largely unknown. Here, we report the cycle shear deformation mechanism of an amorphous polyethylene polymer using molecular dynamics (MD) simulations. The stress-strain behaviors, including the elastic, yield, strain hardening, and strain softening regions, are qualitatively in agreement with the previous results. The values of the yield stress, Young's modulus and ultimate strength obtained from MD simulations are consistent with the previous data. The effects of the shear strain rate, temperature, and cycle shear-loading number on the stress-strain behaviors are investigated. Higher strain rate and a lower temperature result in a higher strength in the amorphous polyethylene polymer, attributed to the agglomeration of high local strains. With the increase of the cycle shear-loading number, the high strain region gradually expands from the upper and lower surface to the interior of the polyethylene polymer matrix, which provides the origin of crack initiation. The energy contributions are used in elucidating the inherent deformation mechanisms within the elastic, yielding, strain hardening, and strain softening regions, and the variation trend of energy is consistent with the stress-strain response.
\end{abstract}

\section{Introduction}

Polymers, due to their good processing performances and excellent mechanical properties, have an important role in industrial materials. The flexibility of tuning a polymer's properties comes from a number of available degrees of freedom: choice of monomers, branching, tacticity, copolymers, blends and composites. ${ }^{1-3}$ Here, the common material polyethylene is selected as the object of study.

Through the computational simulation of polymers, the complex behavior of highly complex polymeric systems can be understood deeply. Coarse grain techniques using united atoms $\mathrm{s}^{\mathbf{4 - 1 2}}$ have been widely used to simulate polymer systems and obtain deformation behaviour at different lengths and time scales. A large number of reports have dealt with the plastic deformation within amorphous polymers, where the shear stress drives plastic deformation in glassy polymers to induce structural change. ${ }^{\mathbf{1 3}}$ For example, shear stress could alter the flexing of dihedral angles, leading to the formation of local

\footnotetext{
${ }^{a}$ State Key Laboratory of Advanced Design and Manufacturing for Vehicle Body, Hunan University, Changsha, 410082, PR China.E-mail: lijia123@hnu.edu.cn

${ }^{b}$ State Key Laboratory of Powder Metallurgy, Central South University, Changsha, 410083, PR China. E-mail: hwucsu@csu.edu.cn

'Shenzhen Nonfemet Technology Co., Ltd, Shenzhen 518122, PR China

${ }^{d}$ School of Materials Science and Engineering, Northwestern Polytechnical University, Xi'an 710072, PR China
}

pockets of liquid-like molecular material. ${ }^{14}$ In line with this notion, shear strain is treated as the accumulation of rotations of kink pairs along the polymer chains and the corresponding model is developed. ${ }^{15}$ In addition, the effect of shear strain on plastic deformation in bulk metallic glasses is revealed during deformation. For instance, by MD simulations, plastic deformation in nanostructure glasses takes place due to local atom rearrangement which produces shear transformation as nucleation sites for additional transformations. ${ }^{16}$ Using molecular statics (MS), the deformation behavior of 3D metallic glasses is studied. ${ }^{17,18}$ In these studies, plastic deformation is due to highly localized heterogeneous atomic rearrangements to generate local stresses. Although studies of atomic/metallic glasses have provided insights into the deformation behavior, the complex bonding of glassy polymers makes their deformation more difficult.

However, so far, numerous computer simulations are performed to study the deformations of glassy polymers in detail. For example, the mechanical deformation of an amorphous linear polyethylene-like polymer glass subjected to uniaxial tension at 100 to $300 \mathrm{~K}$ by atomistic Monte Carlo (MC) simulations is studied..$^{19}$ The stress is firstly elastic and then yields with the increase in strain, and finally strain hardening occurs. In the elastic stage, the mechanical work is mainly translated into the nonbonded internal energy. From the yielding stage forward, the intrachain contributions begin to take effect. For the same strain, the stress is larger at lower temperatures with 
a higher strain rate. ${ }^{19}$ The effect of the constraints and deformation protocol on uniaxial deformation of a polyethylene-like polymer glass by MC simulation is explored. ${ }^{20}$ The study indicates that the constrained bonds and deformation protocol dramatically affect the deformation of polyethylene-like polymer glass, based on the analysis of the stress-strain behavior, evolution of the energy and density, distribution of dihedral angle, and pair correlation function. ${ }^{20}$ The mechanical properties of the polyethylene subjected to uniaxial tension at temperatures of 10 to $500 \mathrm{~K}$ are studied by MD simulation. ${ }^{21}$ The results reveal that the value of the Young's modulus decreases with increasing temperature. At low temperatures, the stressstrain response undergoes elasticity, yield, and plastic flow, but it suffers with the viscoelasticity at high temperatures. ${ }^{21}$ Plastic deformation of a typical flexible chain glassy polymer-polypropylene subjected to pure shear is analyzed. ${ }^{22}$ The results indicate that torsional freedom degrees control the conformational changes, due to the inflexible feature in the backbone bonds and bond angles. ${ }^{22}$ In addition, the mechanical response of glassy polymers is studied via probing the elastic properties of glassy polypropylene using MS techniques. ${ }^{23,24}$

The previous work mentioned above indicates that the plastic deformation of polypropylene depends on the temperature, and applied strain rate. Moreover, it is worth studying the cyclic deformation of polypropylene subjected to homogeneous stress. The effects of the loading rates, temperatures and cycle loading number on the deformation behaviors of amorphous PE is an important issue, however, it is still not well understood during shear loading deformation. In this study, we investigate the elastoplastic properties and deformation behaviors of amorphous polyethylene under shear loading using MD simulations. The effects of shear strain rates and temperature as well as cycle shear-loading number are considered. The deformation mechanisms are shown through describing chain slipping. In addition, the potential energy of amorphous polyethylene is also depicted to explain the elastoplastic deformation behavior.
The paper is organized as follows. The simulated detail is given in Section 2. The results and discussion are described in Section 3. The conclusions based on those observations are summarized in Section 4.

\section{Simulation methodology}

Fig. 1 shows amorphous polyethylene deformed during shear loading. The initial structure of amorphous polyethylene is generated by $\mathrm{C}$ code, as follows: (1) a single perfect polyethylene is produced based on the atomic coordinate relation; (2) other perfect polyethylenes are obtained based on the first polyethylene by changing the atomic coordinate; (3) the formed polyethylenes are set to a system of $800 \mathrm{~K}$ to form the amorphization. Here, the amorphous polyethylene system has large numbers of monomers with 10000 united atoms. The united atoms stand for full atoms (i.e., $\mathrm{CH}_{2}$ ) to improve computational efficiency. ${ }^{25-28}$ The length and number of chains used in amorphous polyethylene are 1000 monomers and 10, respectively. The Dreiding potential, including bond stretching changes, bond-angle changes, dihedral-rotation changes, and van der Waals nonbonded interactions, is applied on the interatomic force field of a united atom model for polyethylene. ${ }^{25-28}$ The respective parameters of the force field in the amorphous polyethylene system are given in the previous work. ${ }^{28}$ Fig. 1b shows the relationship of volume evolution and temperature in polyethylene during the cooling process, where the polyethylene is cooled at a cooling rate of $0.5 \mathrm{~K} \mathrm{ps}^{-1}$ from $500 \mathrm{~K}$ to $0 \mathrm{~K}$ based on the previous work. ${ }^{29}$ By the discontinuity point in the slope between the specific volume and temperature, ${ }^{29}$ a glass transition temperature of $255 \mathrm{~K}$ can be determined in the amorphous polyethylene system, which agrees with the previous results. ${ }^{28,29}$ Hence, the temperatures of 10 , $100,250,400$, and $800 \mathrm{~K}$ are purposely chosen, to study the temperature effect on the shear deformation behaviour.
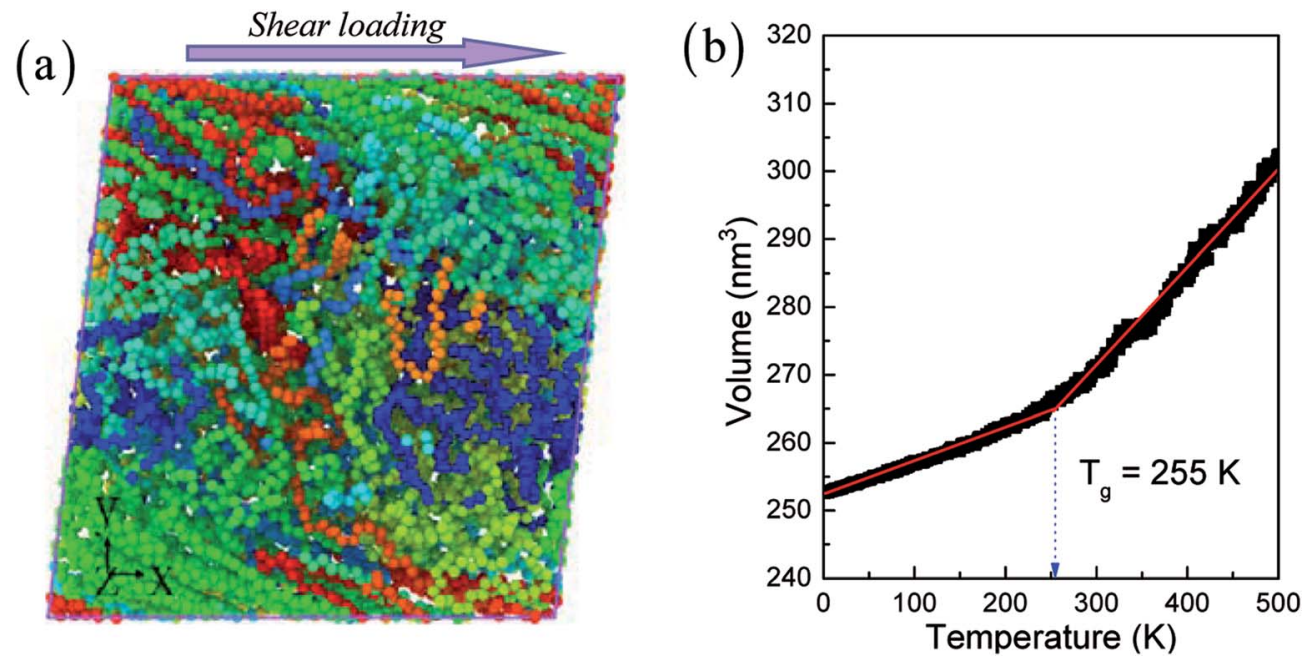

Fig. 1 A deformed configuration of amorphous polyethylene (a). The shear loads are applied along the $x-y$ plane, as indicated by the arrow. The volume vs. temperature in polyethylene during the cooling process (b). 
The periodic boundary conditions are selected along all directions in the current MD simulation, and the time-step is set to $1 \mathrm{fs}$. According to previous work, ${ }^{27,28,30}$ the system of MD simulation is set at $800 \mathrm{~K}$ to generate the amorphous polymer structure, and then cooled to the desired temperature. This relaxation can be achieved by four steps, as follows: (1) firstly, the system of the amorphous polymer is applied to NVT dynamics at $800 \mathrm{~K}$ for $100 \mathrm{ps}$, and then NPT dynamics at $800 \mathrm{~K}$ for $500 \mathrm{ps}$; (2) during the next relaxation, the system of the amorphous polymer is cooled to the desired temperature at a cooling rate of $0.5 \mathrm{~K} \mathrm{ps}^{-1}$, and then is carried out using the NPT ensemble for $500 \mathrm{ps}$ at the desired temperature for relaxing the system and alleviating the out-of-balance forces and net stresses. The system of the amorphous polymer is deformed at the applied shear strain rates between $1 \times 10^{6}$ and $1 \times 10^{9} \mathrm{~s}^{-1}$ under the NPT ensemble with a zero-pressure condition, to investigate the shear strain rate effect using the MD simulation. ${ }^{30-32}$ The large-scale atomic/molecular massively parallel simulator (LAMMPS) ${ }^{33}$ is applied to deform amorphous polyethylene. The open visualization tool OVITO $^{34}$ is used for postprocessing and visualization.

The stresses in different directions are calculated by the average of all atomic stresses, as follows ${ }^{35}$

$$
S=\frac{1}{\Omega} \sum_{i}^{N}\left(m_{i} v_{i} \otimes v_{i}+\frac{1}{2} \sum_{i \neq j} r_{i j} \otimes \frac{\partial U\left(r_{i j}\right)}{\partial r_{i j}}\right)
$$

where $S$ is the average viral stress with six components, $\Omega$ is the volume of the cut-off domain, $m_{i}$ is the mass, $v_{i}$ is the velocity of the atom $i, \otimes$ is the tensor product of two vectors, and $N$ is the total number of atoms in the domain.

\section{Results and discussion}

\subsection{The stress-strain behaviour}

Fig. 2 presents the stress-strain curve and the structures of amorphous polyethylene at different shear strains. According to classical mechanics, the stress-strain curve can be divided into four distinct regimes: elastic, yield, hardening and softening stages $^{36-38}$ (Fig. 2a). The elastic regime is defined as the region which presents the stress to increase linearly with the increasing strain. This trend is consistent with the deformation behavior of metals. ${ }^{37}$ The stress increases nearly linearly with the increase of shear strain, indicating an elastic deformation. After the yielding, the stress continually increases to a local maximum, suggesting the material hardens. After the hardening stage, the stress reduces with the increase of strain, showing the softening stage in the stress-strain curve. Similar behavior is reported in the plastic deformation of amorphous polyethylene using MD simulations. ${ }^{28,29,39}$

Young's modulus, yield stress, and ultimate strength are the important characterizations of the mechanical properties of materials. The Young's modulus is defined as $\sigma / \varepsilon$, where $\sigma$ is the stress, and $\varepsilon$ is the strain. The yield peak is defined as the maximum stress when the initial plastic deformation just occurs. The ultimate strength is defined as the maximum stress in the stress-strain curve. Fig. 2a indicates that the values of the yield stress, Young's modulus and ultimate strength are $130 \mathrm{MPa}, 2.8 \mathrm{GPa}$ and $224 \mathrm{MPa}$, respectively. These results are in good agreement with the yield stress of $152 \mathrm{MPa}$, the Young's modulus of $2.5 \mathrm{GPa}$, and the ultimate strength of $232 \mathrm{MPa}$ in the

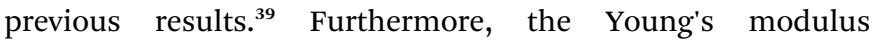
computed by MD simulations is consistent with an experimental range of between 2.24 and $3.8 \mathrm{GPa} .^{40}$ The corresponding
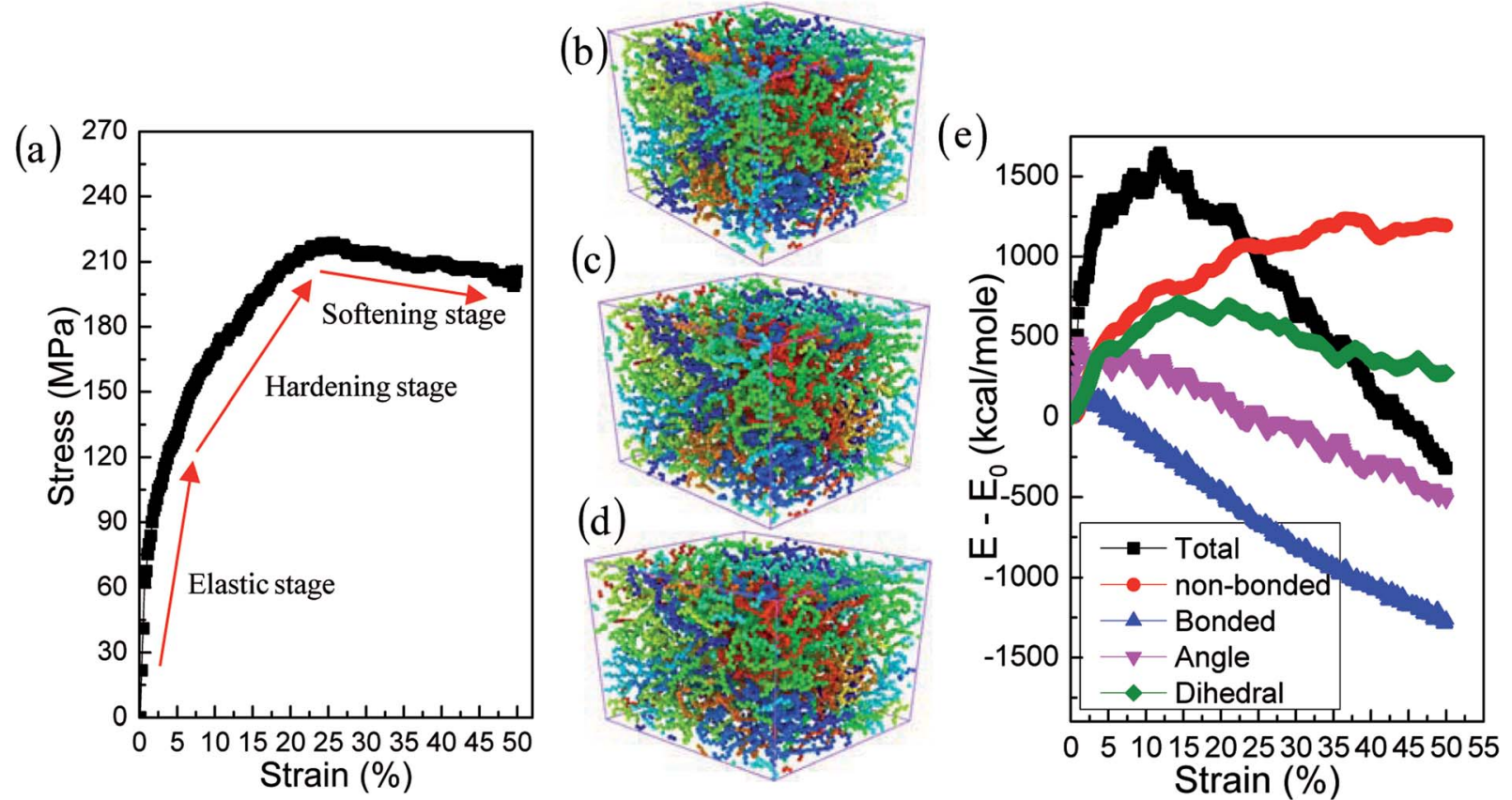

Fig. 2 Shear stress versus shear strain curves at the shear strain rate of $1 \times 10^{9} \mathrm{~s}^{-1}$ and a temperature of $250 \mathrm{~K}$ (a). The structures of amorphous polyethylene at the shear strain of $30 \%$ (b), $40 \%$ (c), and 50\% (d). Energy decomposition of an amorphous polyethylene system (e). 
(a)

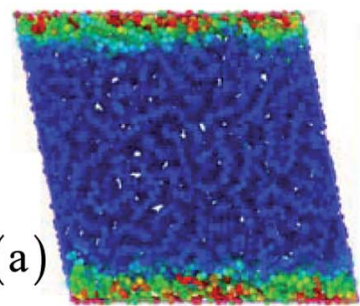

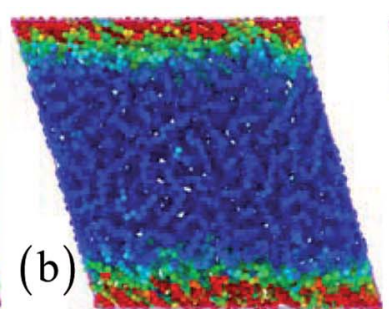
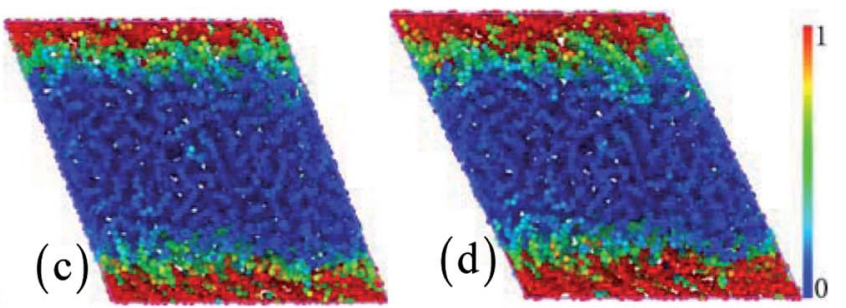

Fig. 3 Distributions of local atom shear strain at the shear strain rate of $1 \times 10^{9} \mathrm{~s}^{-1}$, the temperature of $250 \mathrm{~K}$, and different strains of $20 \%$ (a), $30 \%$ (b), $40 \%$ (c), and $50 \%$ (d).

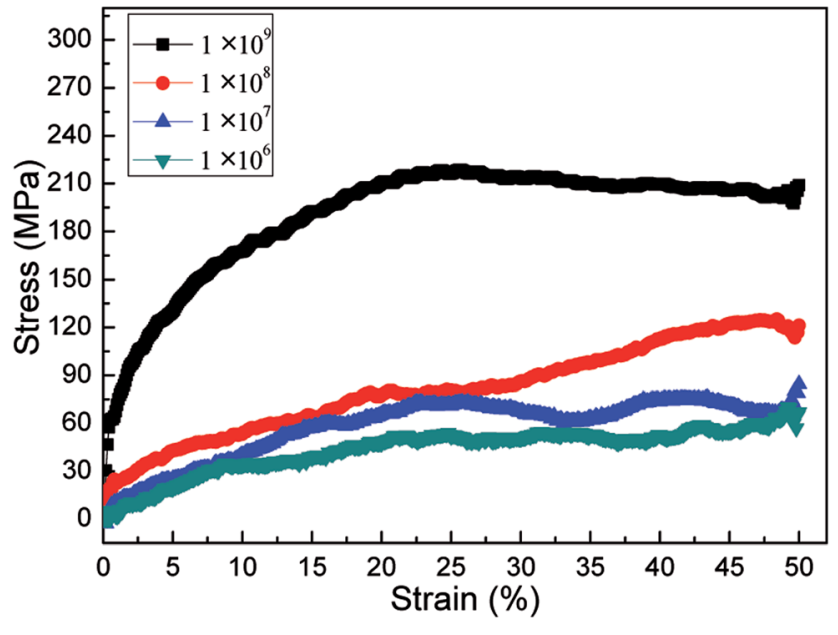

Fig. 4 Stress-strain response of amorphous polyethylene deformed at the temperature of $250 \mathrm{~K}$ and different strain rates: $1 \times 10^{6}, 1 \times 10^{7}$ $1 \times 10^{8}$, and $1 \times 10^{9} \mathrm{~s}^{-1}$. deformation structures are shown in Fig. $2 \mathrm{~b}-\mathrm{d}$, at the strain of $30 \%, 40 \%$, and $50 \%$. Fig. 2e illustrates the change of the total energy, the non-bonding energy, the bonding energy, the bond angle energy and the dihedral energy in amorphous polyethylene at the temperature of $250 \mathrm{~K}$ and the strain rate of $1 \times$ $10^{9} \mathrm{~s}^{-1}$. With the increase of shear strain, the dihedral and nonbonded energies increase sharply at the elastic, yield, and hardening stages, due to chain slippage. When the strain is larger than $25 \%$, the total energy reduces sharply, consistent with the stress-strain response in Fig. 2a. To deeply understand the strain effect, the local atomic shear strain distribution to measure the local inelastic deformation determines the damage location. ${ }^{\mathbf{4 1}}$ The distributions of shear strain are plotted in Fig. 3 at different strains. The high strain regions spread from surface to interior, indicating the increase of damage.

\subsection{Shear strain rate effect}

The effects of strain rate on the structural response and mechanical properties of amorphous polyethylene under $250 \mathrm{~K}$ are discussed. Fig. 4 shows the relationship of stress and strain
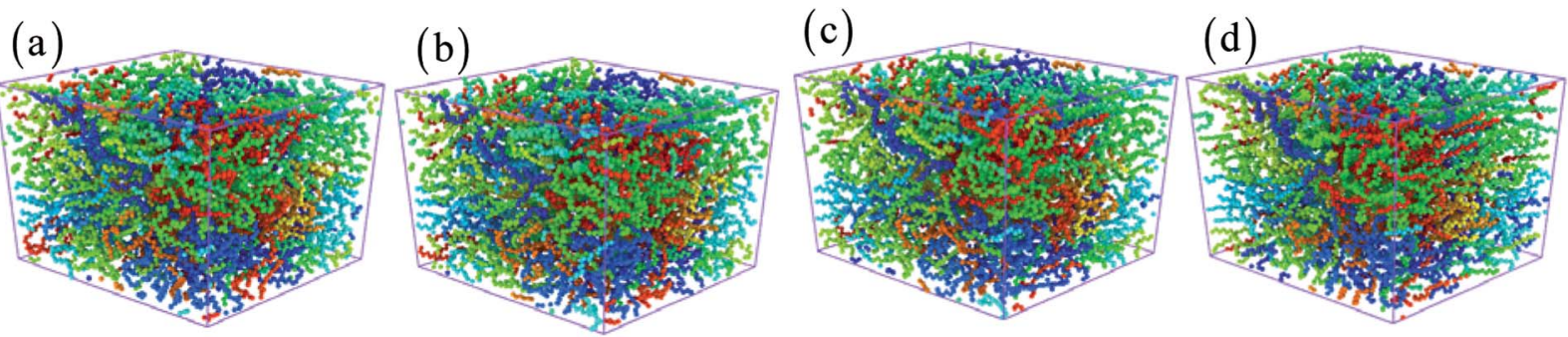

Fig. 5 The structure of amorphous polyethylene at the temperature of $250 \mathrm{~K}$ and different strain rates: $1 \times 10^{6}$ (a), $1 \times 10^{7}$ (b), $1 \times 10^{8}$ (c), and $1 \times$ $10^{9} \mathrm{~s}^{-1}(\mathrm{~d})$.
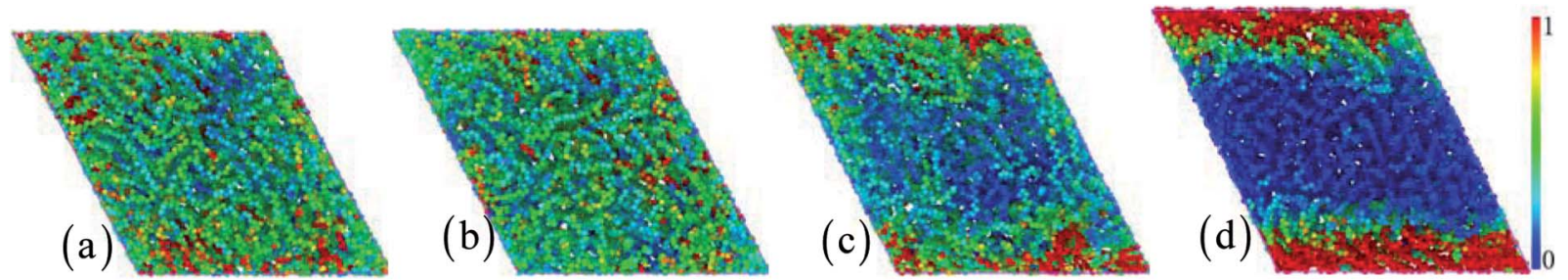

Fig. 6 Local atom shear strain distribution at the temperature of $250 \mathrm{~K}$ and different strain rate: $1 \times 10^{6}$ (a), $1 \times 10^{7}$ (b), $1 \times 10^{8}$ (c), and $1 \times 10^{9} \mathrm{~s}^{-1}$ (d). 


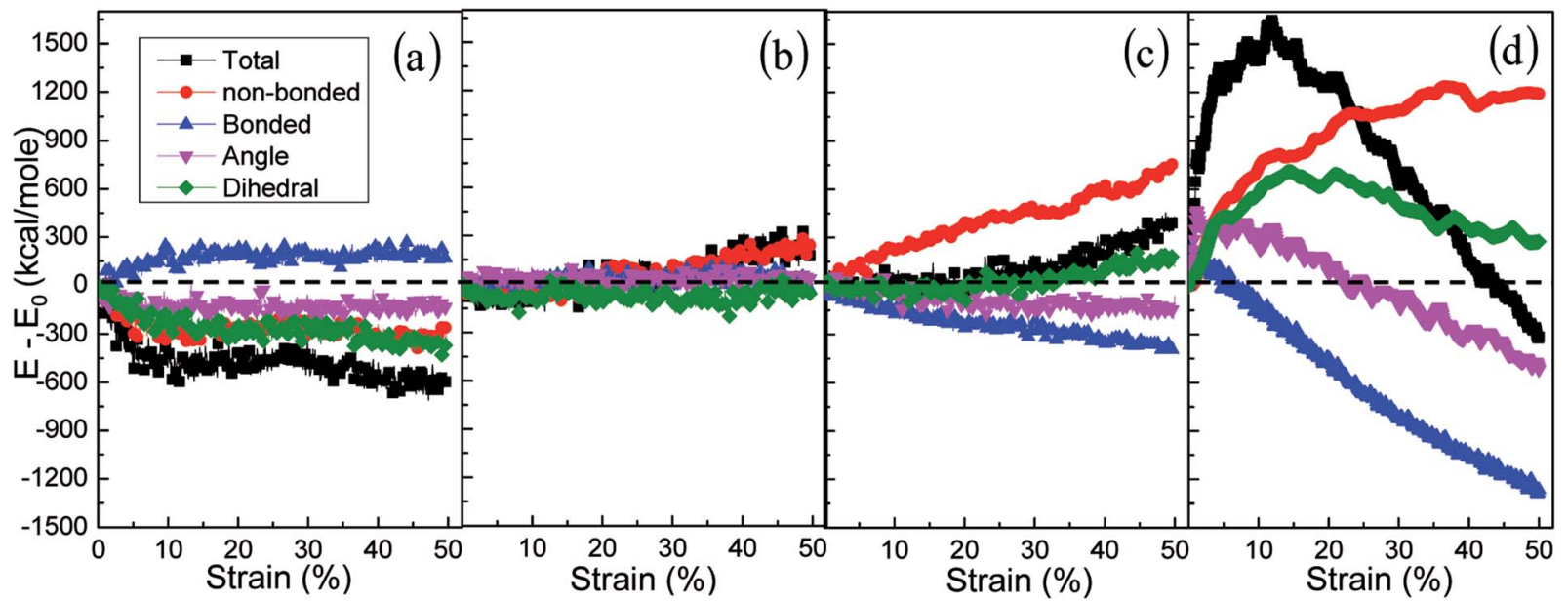

Fig. 7 Energy decomposition of an amorphous polyethylene system at the temperature of $250 \mathrm{~K}$ and different strain rates: $1 \times 10^{6}(\mathrm{a}), 1 \times 10^{7}$ (b), $1 \times 10^{8}(\mathrm{c})$, and $1 \times 10^{9} \mathrm{~s}^{-1}(\mathrm{~d})$.

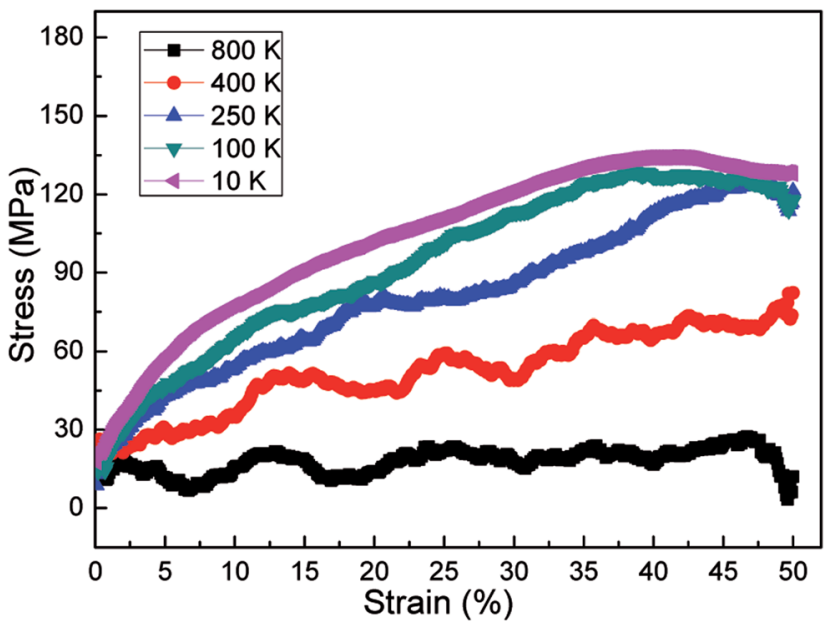

Fig. 8 Stress-strain response of amorphous polyethylene deformed at the strain rate of $1 \times 10^{8} \mathrm{~s}^{-1}$, and different temperatures of 10,100 , 250,400 , and $800 \mathrm{~K}$.

in amorphous polyethylene at different strain rates. The yielding strength decreases sharply with the decrease of strain rate, which agrees with the previous results. ${ }^{42,43}$ It suggests that the response of stress and strain is sensitive to the shear strain rate, especially for the high shear strain rate. Fig. 5 shows snapshots of amorphous polyethylene under a shear strain of $50 \%$ for different strain rates. It can be seen that the amorphous polyethylene subjected to shear loading deforms by increasing chain length. After shear deformation, the atomic strain distributions at various shear strain rates are shown in Fig. 6. At a low shear strain rate the local strain is evenly distributed, however, at a high shear strain rate the high local strain is located at the end of amorphous polyethylene. This indicates that the microstructural evolution of amorphous polyethylene controls the stress trend (see Fig. 4). In addition, the low strain occurs in the middle region and there is no obvious change in the chain of amorphous polyethylene at high strain rate, leading to the stress oscillation in Fig. 4. The variation in internal energy as a function of strain rate for the amorphous polyethylene system at $250 \mathrm{~K}$ is shown in Fig. 7. Here, the energy evolution depends on the applied strain rate, which spans three orders of magnitude. At the low strain rate of $1 \times 10^{6} \mathrm{~s}^{-1}$, the trends of the total energy and non-bonded interaction energy are very different, compared to that at the high strain rate of $1 \times$ $10^{7} \mathrm{~s}^{-1}$. At a strain rate of $1 \times 10^{7} \mathrm{~s}^{-1}$, the energies associated with bond length and bond angles change slightly with an increase of strain, and the nonbonded interaction energy increases slightly with increasing strain. (a)

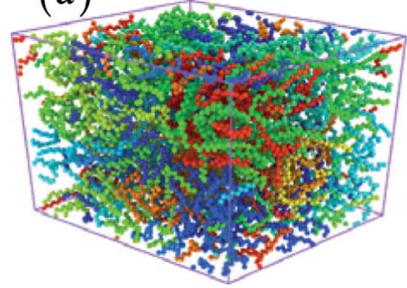

(b)

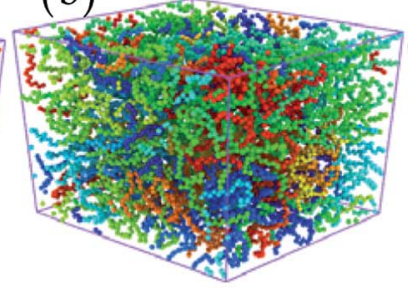

(c)

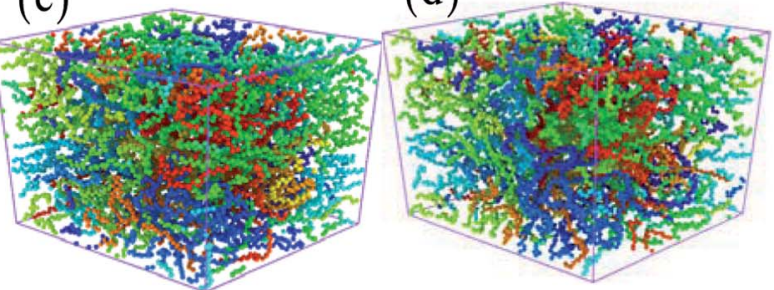

Fig. 9 The structure of amorphous polyethylene at the strain rate of $1 \times 10^{8} \mathrm{~s}^{-1}$, and different temperatures of 10 (a), 100 (b), 400 (c), and $800 \mathrm{~K}$ (d). 

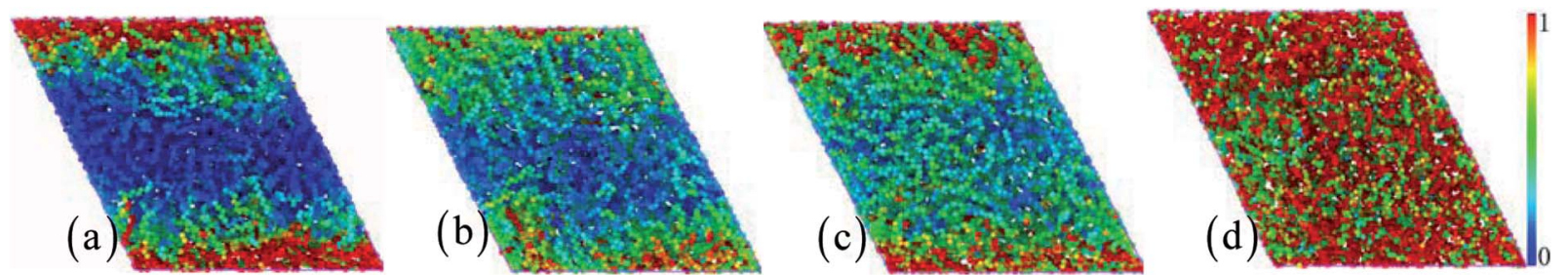

Fig. 10 The local atom shear strain distribution of amorphous polyethylene at the strain rate of $1 \times 10^{8} \mathrm{~s}^{-1}$, and different temperatures of 10 (a), 100 (b), 400 (c), and $800 \mathrm{~K}(\mathrm{~d})$

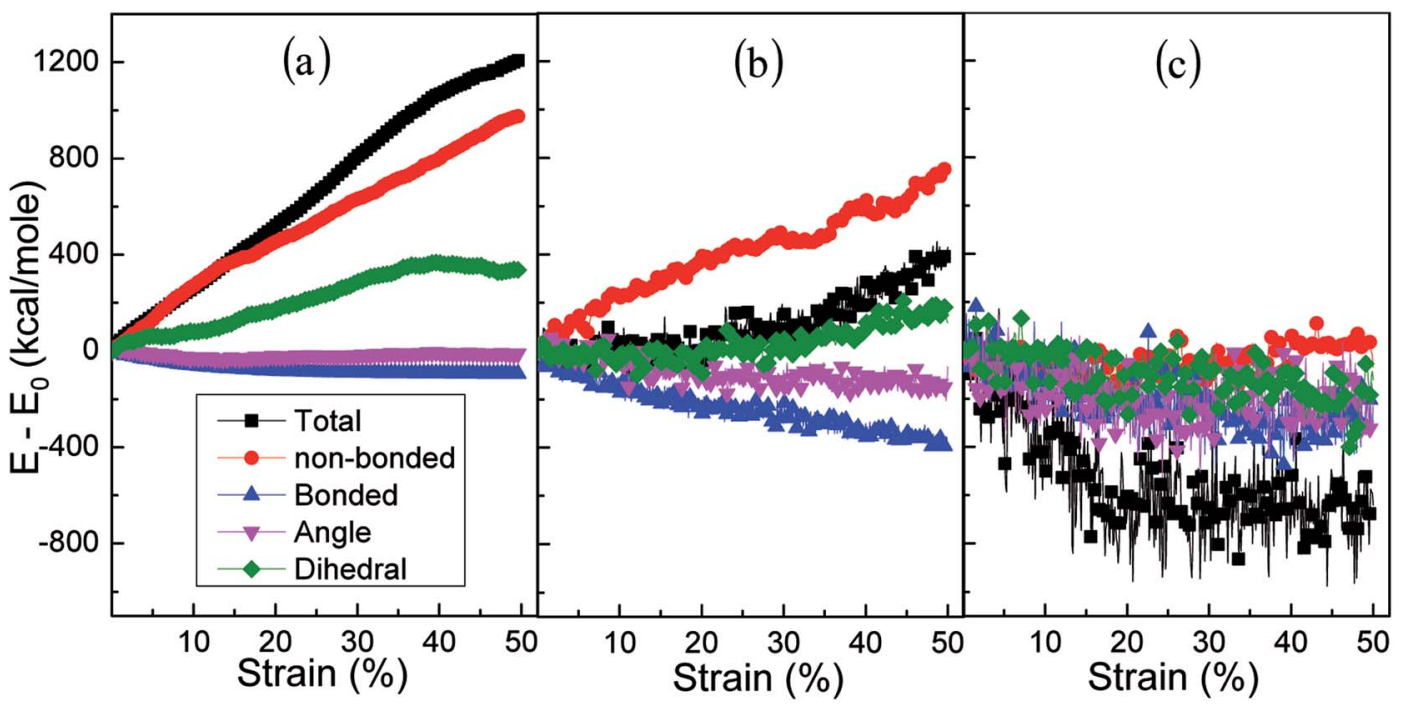

Fig. 11 Energy decomposition of an amorphous polyethylene system at the strain rate of $1 \times 10^{8} \mathrm{~s}^{-1}$, and different temperatures of 10 (a), 250 (b), and $800 \mathrm{~K}(\mathrm{c})$

\subsection{Temperature effect}

Temperature plays a key role in the stress-strain response and microstructural evolution of amorphous polyethylene. ${ }^{\mathbf{4 4 , 4 5}}$ In Fig. 8, the high temperature can soften the amorphous polyethylene, and the low temperature can strengthen the amorphous polyethylene during shear deformation, similar to the mechanical behaviour of metals at different temperatures. The shear stress of amorphous polyethylene fluctuates when the temperature is larger than $100 \mathrm{~K}$. The structure and local atom shear strain distribution of amorphous polyethylene at the strain of $50 \%$ are displayed in Fig. 9 and 10. The high temperature produces the high strain of amorphous polyethylene, to

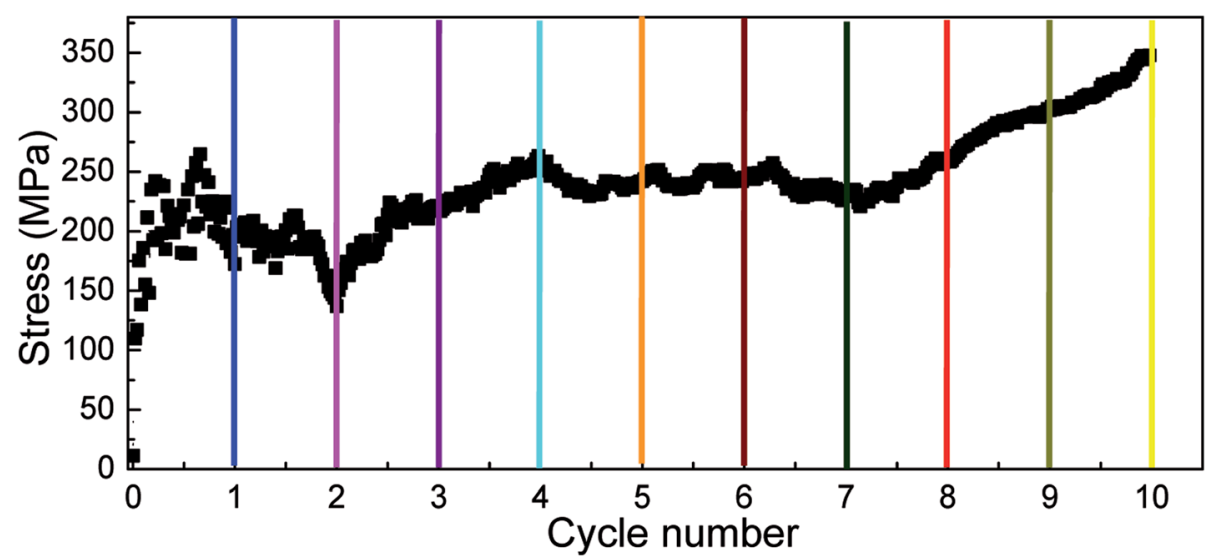

Fig. 12 Stress response of amorphous polyethylene deformed at the strain rate of $1 \times 10^{9} \mathrm{~s}^{-1}$, a temperature of $250 \mathrm{~K}$, and different cycle shearloading numbers. One shear-loading cycle is defined as the system subjected to one cycle of shear deformation and reverse shear deformation. 
(a)

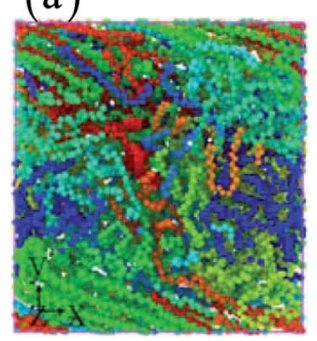

(b)

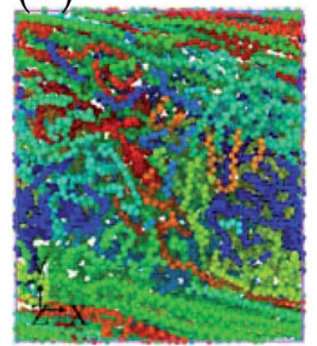

(c)

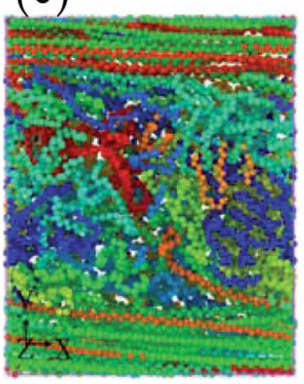

(d)

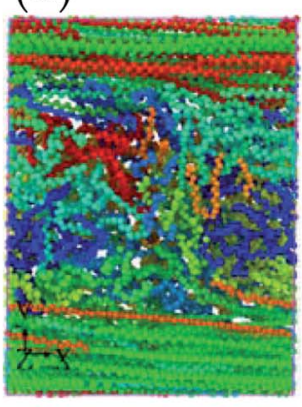

(e)

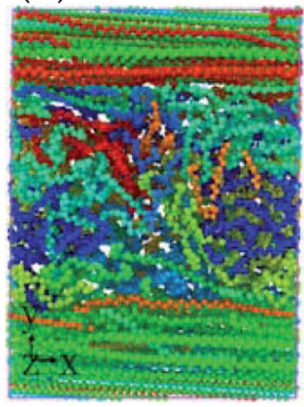

Fig. 13 The structure of amorphous polyethylene at the strain rate of $1 \times 10^{9} \mathrm{~s}^{-1}$, the temperature of $250 \mathrm{~K}$, and different cycle numbers of 1 (a), 2 (b), 5 (c), 7 (d), and 10 (e).

(a)

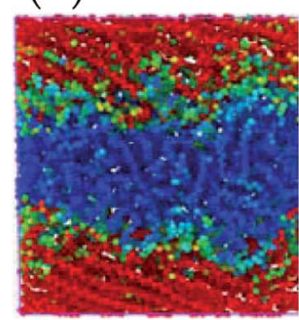

(b)

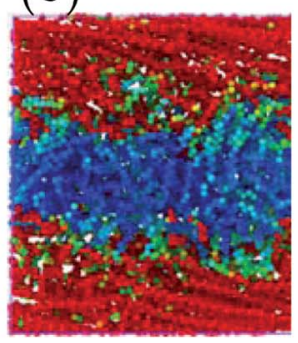

(c)

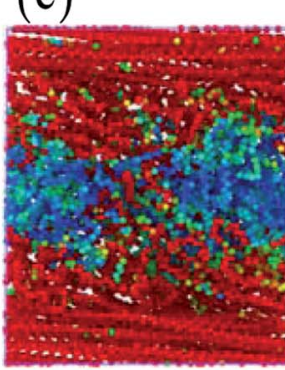

(d)

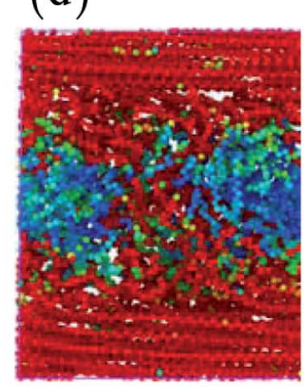

(e)

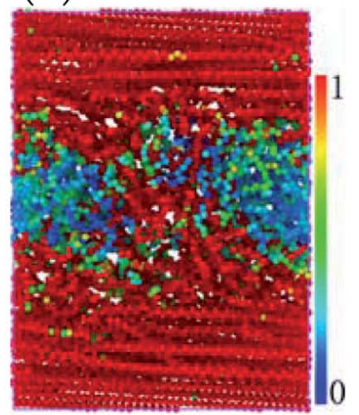

Fig. 14 The local atom shear strain distribution of amorphous polyethylene at the strain rate of $1 \times 10^{9} \mathrm{~s}^{-1}$, the temperature of $250 \mathrm{~K}$, and different cycle numbers of 1 (a), 2 (b), 5 (c), 7 (d), and 10 (e).

reduce the shear stress. The low temperature inhibits the chain slippage, and the high strain takes place in the small region, resulting in the decrease of the stress fluctuation (see Fig. 4). Fig. 11 shows the change in energy decomposition of an amorphous polyethylene system at different temperatures. The general trend is that the energies associated with bond length and bond angles change obviously with the increase of strain at a wide range from 10 to $800 \mathrm{~K}$. At the high temperature of $800 \mathrm{~K}$, all energies always reduce as the shear strain increases. However, at low temperatures of 10 and $250 \mathrm{~K}$, the total energy, the non-bonded energy, and the dihedral energy increase with the increase of shear strain. The results show that the temperature has a significant effect on the energy evolution, especially at a low temperature of $10 \mathrm{~K}$.

\subsection{Cycle shear-loading number effect}

The cyclic shear loading is applied to the amorphous polyethylene, ${ }^{46,47}$ to study the fatigue behavior. The stress sharply fluctuates at cycle numbers below 3 , and then keeps stable at cycle numbers from 4 to 8 , finally increasing continuously at cycle numbers larger than 9, as shown in Fig. 12. With increasing cycle number, the structure and local atom shear strain distribution of amorphous polyethylene are plotted in Fig. 13 and 14. The chain becomes straight and smooth at the end of amorphous polyethylene, as the cycle number increases.
In addition, this trend gradually expands towards the inside of amorphous polyethylene in Fig. 13. At high cycle numbers, a similar shear band occurs through the interior of amorphous polyethylene, resulting in an increase of shear stress. Hence, the evolution of the local atom shear strain distribution as a function of cycle number can provide insight into the accommodation of deformations in amorphous polyethylene. Fig. 15 shows how the energy of amorphous polyethylene evolves as a function of cycle shear number at $250 \mathrm{~K}$. With increasing cycle shear number, the non-bonding energy and the dihedral energy play a weak role in the deformation behaviour. The total energy increases with the increasing strain at high cycle shear-loading numbers, unlike the behavior at the low cycle shear-loading number. Interestingly, all energies change slowly at a middle cycle number due to chain slipping.

Cyclic shear loading with high cycle numbers would result in the fracture of amorphous polyethylene. The strain to the internal amorphous polyethylene increases with the increase of cycle number (see Fig. 14), and finally the cycle maximal stress always exceeds its rupture strength to cause serious failure. In addition, the size of the amorphous polyethylene system strongly affects the fracture mechanism, owing to the size effects on the fracture energy observed in the experiment of polymer fracture. ${ }^{48,49}$ The MD simulations of the fracture could give a fundamental understanding of the solids failure, 


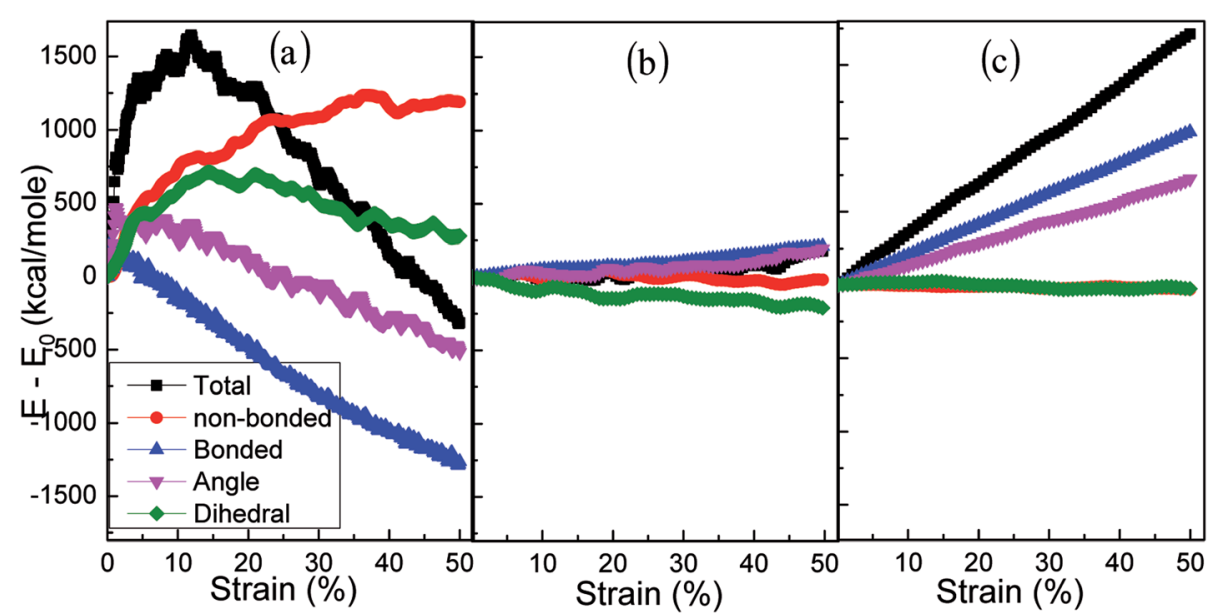

Fig. 15 Energy decomposition of an amorphous polyethylene system at the strain rate of $1 \times 10^{9} \mathrm{~s}^{-1}$, a temperature of $250 \mathrm{~K}$, and different cycle numbers of 1 (a), 5 (b), and 10 (c).

however, MD simulations are restricted to a certain size and time in the amorphous polyethylene. Recently, the most important development of the multiscale modeling of the fracture or modeling fracture would propose new solutions for the multiscale fracture process..$^{50,51}$ Multiscale methods coupling MD-models to continuum models ${ }^{50,51}$ have been developed, which are conducive to investigating the behaviour of multiscale fracture in the amorphous polyethylene. In future, to deal with the uncertainties of fracture toughness in the amorphous polyethylene, we would use a sensitivity analysis toolbox to quantify the influence of different parameters on uncertain fracture toughness..$^{52}$ The accurate prediction of fracture strength could help to optimize reasonably the design structure of the amorphous polyethylene for industrial applications.

\section{Conclusions}

In the present study, the shear deformations of amorphous polyethylene are investigated by MD simulations. The effects of temperature, shear strain rate, and cycle shear-loading number on the deformation and local atom shear strain distribution in amorphous polyethylene are analyzed in terms of the energy evolution and the stress-strain behaviour. The mechanical properties, including the yield stress, Young's modulus, and ultimate strength, in the amorphous polyethylene can be obtained from MD simulations, which are in quantitative agreement with previous results. The MD simulation reveals that the strength of amorphous polyethylene can be improved by increasing the shear strain rate, or lowering the temperature. The shear strain rate, temperature, and cycle shear-loading number play a key role in the deformation, local atom shear strain distribution and energy evolution. The current study is beneficial for deeply understanding the deformation and strengthening mechanisms of the amorphous polyethylene in complex external environments, and could provide a new study basis for searching for high-performance amorphous polyethylene applied in extreme environments.

\section{Conflicts of interest}

The authors declare no conflict of interest.

\section{Acknowledgements}

The authors deeply appreciate the support from the NNSFC (11572118, 11772122, 51771233, and 51871092), the Fundamental Research Funds for the Central Universities (531107051151), China Postdoctoral Science Foundation (Grant No. 2018M633164), Hunan Natural Science Foundation (2018JJ3651), the Hunan Youth Science and Technology Innovation Talent Program, and the National Key Research and Development Program of China (2016YFB0700300 and 2016YFB1100103).

\section{References}

$1 \mathrm{~J}$. Nicholson, The chemistry of polymers, Royal Society of Chemistry, 2017.

2 S. Laurichesse and L. Averous, Chemical modification of lignins: Towards biobased polymers, Prog. Polym. Sci., 2014, 39, 1266-1290.

3 D. Venkateshvaran, M. Nikolka, A. Sadhanala, V. Lemaur, M. Zelazny, M. Kepa and I. Romanov, Approaching disorder-free transport in high-mobility conjugated polymers, Nature, 2014, 515, 384-391.

4 S. Deng, A. Arinstein and E. Zussman, Size-dependent mechanical properties of glassy polymer nanofibers via molecular dynamics simulations, J. Polym. Sci., Part B: Polym. Phys., 2017, 55(6), 506-514.

$5 \mathrm{~W}$. G. Noid, Perspective: Coarse-grained models for biomolecular systems, J. Chem. Phys., 2013, 139, 090901090925.

6 F. Baset, K. Popov, A. Villafranca, A. M. Alshehri, J. M. Guay, L. L. Ramunno and V. R. Bhardwaj, Nanopillar formation from two-shot femtosecond laser ablation of poly-methyl methacrylate, Appl. Surf. Sci., 2015, 357, 273-281. 
7 K. M. Salerno, A. Agrawal, D. Perahia and G. S. Grest, Resolving dynamic properties of polymers through coarsegrained computational studies, Phys. Rev. Lett., 2016, 116, 058302-058310.

8 T. Y. Molotilin, S. R. Maduar and O. I. Vinogradova, Star polymers as unit cells for coarse-graining cross-linked networks, Phys. Rev. E, 2018, 97, 032504-032512.

9 K. K. Bejagam and S. Balasubramanian, Supramolecular polymerization: a coarse grained molecular dynamics study, J. Phys. Chem. B, 2015, 119, 5738-5746.

10 M. A. C. Stuart, W. T. Huck, J. Genzer, M. Müller, C. Ober, M. Stamm and F. Winnik, Emerging applications of stimuli-responsive polymer materials, Nat. Mater., 2010, 9, 101-109.

11 W. Brostow, S. Khoja and R. Simoes, Sliding wear behavior of polymers studied with mesoscopic molecular dynamics, $J$. Mater. Sci., 2017, 52, 1203-1213.

12 Q. H. Zeng, A. B. Yu and G. Q. Lu, Multiscale modeling and simulation of polymer nanocomposites, Prog. Polym. Sci., 2008, 33, 191-269.

13 K. B. Migler, H. Hervet and L. Leger, Slip transition of a polymer melt under shear stress, Phys. Rev. Lett., 1993, 70, 287-294.

14 M. D. Wang, E. Nakanishi and S. Hibi, Finite element analysis of single-edgecracked rolled high-density polyethylene, Polymer, 1994, 35, 1442-1451.

15 G. Z. Voyiadjis, A. Shojaei and G. Li, A generalized coupled viscoplastic-viscodamage-viscohealing theory for glassy polymers, Int. J. Plast., 2012, 28, 21-45.

16 E. Bouchbinder, J. S. Langer and I. Procaccia, Athermal shear-transformation-zone theory of amorphous plastic deformation. I. Basic principles, Phys. Rev. E, 2007, 75, 036107-036115.

17 T. Brink, L. Koch and K. Albe, Structural origins of the boson peak in metals: From high-entropy alloys to metallic glasses, Phys. Rev. B: Condens. Matter Mater. Phys., 2016, 94, 224203224211.

18 T. Brink, M. Peterlechner, H. Rösner, K. Albe and G. Wilde, Influence of crystalline nanoprecipitates on shear-band propagation in Cu-Zr-based metallic glasses, Phys. Rev. Appl., 2016, 5, 054005-054012.

19 J. Li, T. Mulder, B. Vorselaars, A. V. Lyulin and M. A. Michels, J. Monte Carlo simulation of uniaxial tension of an amorphous polyethylene-like polymer glass, Macromolecules, 2006, 39, 7774-7782.

20 T. Mulder, J. Li, A. V. Lyulin and M. A. Michels, Monte carlo simulation of uniaxial deformation of polyethylene-like polymer glass: role of constraints and deformation protocol, Macromol. Theory Simul., 2007, 16, 348-358.

21 D. Brown and J. H. Clarke, Molecular dynamics simulation of an amorphous polymer under tension. 1. Phenomenology, Macromolecules, 1991, 24, 2075-2082.

22 A. S. Argon, P. H. Mott and U. W. Suter, Simulation of plastic deformation in a flexible chain glassy polymer, Phys. Status Solidi B, 1992, 172, 193-204.

23 A. Weerasinghe, C. T. Lu, D. Maroudas and A. Ramasubramaniam, Multiscale Shear-Lag Analysis of
Stiffness Enhancement in Polymer-Graphene Nanocomposites, ACS Appl. Mater. Interfaces, 2017, 9, 23092-23098.

24 G. Molnár, P. Ganster, A. Tanguy, E. Barthel and G. Kermouche, Densification dependent yield criteria for sodium silicate glasses-An atomistic simulation approach, Acta Mater., 2016, 111, 129-137.

25 F. M. Capaldi, M. C. Boyce and G. C. Rutledge, Enhanced mobility accompanies the active deformation of a glassy amorphous polymer, Phys. Rev. Lett., 2002, 89, 175505175512.

26 F. M. Capaldi, M. C. Boyce and G. C. Rutledge, Molecular response of a glassy polymer to active deformation, Polymer, 2004, 45, 1391-1399.

$27 \mathrm{C}$. $\mathrm{Wu}$ and $\mathrm{W}$. Xu, Atomistic molecular modelling of crosslinked epoxy resin, Polymer, 2006, 47, 6004-6009.

28 D. Hossain, M. A. Tschopp, D. K. Ward, J. L. Bouvard, P. Wang and M. F. Horstemeyer, Molecular dynamics simulations of deformation mechanisms of amorphous polyethylene, Polymer, 2010, 51, 6071-6083.

29 N. Vu-Bac, T. Lahmer, H. Keitel, J. Zhao, X. Zhuang and T. Rabczuk, Stochastic predictions of bulk properties of amorphous polyethylene based on molecular dynamics simulations, Mech. Mater., 2014, 68, 70-84.

30 J. M. Kim, R. Locker and G. C. Rutledge, Plastic deformation of semicrystalline polyethylene under extension, compression, and shear using molecular dynamics simulation, Macromolecules, 2014, 47, 2515-2528.

31 Y. Tian, Z. Li and K. Cai, Wrinkling behaviour of annular graphynes under circular shearing load using molecular dynamics simulations, Nanomater. Nanotechnol., 2015, 5, 9-16.

32 Z. Zheng, Z. Wang, L. Wang, J. Liu, Y. Wu and L. Zhang, Dispersion and shear-induced orientation of anisotropic nanoparticle filled polymer nanocomposites: insights from molecular dynamics simulation, Nanotechnology, 2016, 27, 265704-265711.

33 S. Plimpton, Fast parallel algorithms for short-range molecular dynamics, J. Comp. Physiol., 1995, 117, 1-19.

34 A. Stukowski, Visualization and analysis of atomistic simulation data with OVITO-the Open Visualization Tool, Model. Numer. Simul. Mater. Sci., 2009, 18, 015012-015021.

35 H. Heinz, W. Paul and K. Binder, Calculation of local pressure tensors in systems with many-body interactions, Phys. Rev. E, 2005, 72, 066704-066715.

36 Z. Qin and M. J. Buehler, Molecular dynamics simulation of the $\alpha$-helix to $\beta$-sheet transition in coiled protein filaments: Evidence for a critical filament length scale, Phys. Rev. Lett., 2010, 104, 198304-198311.

37 P. Wang, X. Yang and D. Peng, Molecular dynamics investigation of the grain boundary migration hysteresis of nanocrystalline Ni under cyclic shear loading, Model. Numer. Simul. Mater. Sci., 2016, 25, 025006-025013.

38 X. Lu, X. Guo, J. Yin, Y. Wei, X. Nan, Q. Dong and P. La, Shear mechanical property of $\beta-\mathrm{Si}_{3} \mathrm{~N}_{4}$ nano-thin layers in the basal plane using molecular dynamics simulations, J. Mater. Sci. Eng. A, 2015, 648, 72-79. 
39 F. M. Capaldi, M. C. Boyce and G. C. Rutledge, Enhanced mobility accompanies the active deformation of a glassy amorphous polymer, Phys. Rev. Lett., 2002, 89, 175505175509.

$40 \mathrm{Y}$. Han and J. Elliott, Molecular dynamics simulations of the elastic properties of polymer/carbon nanotube composites, Comput. Mater. Sci., 2007, 39, 315-323.

41 F. Shimizu, S. Ogata and J. Li, Theory of shear banding in metallic glasses and molecular dynamics calculations, Mater. Trans., 2007, 48, 2923-2927.

42 S. Shadlou, B. Ahmadi-Moghadam and F. Taheri, The effect of strain-rate on the tensile and compressive behavior of graphene reinforced epoxy/nanocomposites, Mater. Des., 2014, 59, 439-447.

43 P. Laurienzo, B. Immirzi and M. Malinconico, A preliminary investigation on the use of poly copolymer as compatibiliser of hdpe/pet blends, Macromol. Mater. Eng., 2001, 286, 248253.

44 L. Lan, J. Wu, Y. Yin, X. Li and Z. Li, Effect of temperature on space charge trapping and conduction in cross-linked polyethylene, IEEE Trans. Dielectr. Electr. Insul., 2014, 21, 1784-1791.

45 M. Perumalsamy and T. Murugesan, Phase compositions, molar mass, and temperature effect on densities, viscosities, and liquid-liquid equilibrium of polyethylene glycol and salt-based aqueous two-phase systems, J. Chem. Eng. Data, 2009, 54, 1359-1366.
46 A. Salazar, J. Rodríguez, F. Arbeiter, G. Pinter and A. B. Martínez, Fracture toughness of high density polyethylene: Fatigue pre-cracking versus femtolaser, razor sharpening and broaching, Eng. Fract. Mech., 2015, 149, 199-213.

47 S. Deveci and D. Fang, Correlation of molecular parameters, strain hardening modulus and cyclic fatigue test performances of polyethylene materials for pressure pipe applications, Polym. Test., 2017, 62, 246-253.

48 P. Luna, C. Bernal, A. Cisilino, P. Frontini, B. Cotterell and Y. W. Mai, The application of the essential work of fracture methodology to the plane strain fracture of ABS 3-point bend specimens, Polymer, 2003, 44, 1145-1150.

49 S. Hashemi, Work of fracture of high impact polystyrene (HIPS) film under plane stress conditions, J. Mater. Sci., 2003, 38, 3055-3062.

50 H. Talebi, M. Silani, S. P. Bordas, P. Kerfriden and T. Rabczuk, A computational library for multiscale modeling of material failure, Comput. Mech., 2014, 53, 1047-1071.

51 H. Talebi, M. Silani and T. Rabczuk, Concurrent multiscale modeling of three dimensional crack and dislocation propagation, Adv. Eng. Softw., 2015, 80, 82-92.

52 N. Vu-Bac, T. Lahmer, X. Zhuang, T. Nguyen-Thoi and T. Rabczuk, A software framework for probabilistic sensitivity analysis for computationally expensive models, Adv. Eng. Softw., 2016, 100, 19-31. 